\title{
Methodology in the Mirror: a Living, Systematic Review of Works in Quantitative Ethnography
}

S. Zörgő, GJY. Peters, C. Porter, M. Moraes, S. Donegan, B. Eagan

This paper has been published in the International Conference on Quantitative Ethnography (2021). Citation information is:

2022 Zörgő, S., Peters, GJY., Porter, C., Moraes, M., Donegan, S., Eagan, B. Methodology in the Mirror: a Living, Systematic Review of Works in Quantitative Ethnography. In: Advances in Quantitative Ethnography. Communications in Computer and Information Science, Vol 1522. Eds. Wasson B and Zörgö S., pp 144-159. Cham, Switzerland: Springer Nature. 


\title{
Methodology in the Mirror: a Living, Systematic Review of Works in Quantitative Ethnography
}

\author{
${ }_{\text {S. Zörgö1 [0000-0002-6916-2097] }}{ }^{\text {[0 GJY. Peters }}{ }^{2 \text { [0000-0002-0336-9589] }}$, C. Porter ${ }^{3 \text { [0000-0002- }}$ \\ 6098-5003], M. Moraes ${ }^{4}$ [0000-0002-9652-3011], S. Donegan 3 , B. Eagan 3 [0000-0002-9189-1747] \\ ${ }^{1}$ Care and Public Health Research Institute, Maastricht University, Netherlands \\ zorgoszilvia@gmail.com \\ 2 Faculty of Psychology, Open University, Netherlands \\ ${ }^{3}$ University of Wisconsin-Madison, Madison, WI 53706, USA \\ ${ }^{4}$ Colorado State University, Fort Collins, CO 80542, USA
}

\begin{abstract}
Quantitative Ethnography is a nascent field now formulating the specifics of its conceptual framework and terminology for a unified, quantitative qualitative methodology. Our living, systematic review aims to shed light on decisions in research design that the community has made thus far in the domain of data collection, coding \& segmentation, analysis, and how Quantitative Ethnography as a methodology is conceptualized. Our analysis intends to spur discussions on these issues within the community and help establish a lingua franca.
\end{abstract}

Keywords: Systematic Review · Quantitative Ethnography · Methodology

\section{Introduction}

Quantitative ethnography (QE) is a nascent field aiming to establish a unified, quantitative - qualitative research methodology. This endeavor entails the formulation of novel concepts, negotiation of terminology, and developing new techniques that serve a wide variety of research initiatives in multiple disciplines. There are some key hubs contributing to this process, but it is by and large a polycentric effort; currently circa 400 researchers from over 130 institutions in at least 30 countries engage with QE and the QE community to test and generate theories, implement theoretical constructs, and refine existing knowledge. Due to the interdisciplinary nature of QE, this conceptual framework needs to be traversable by researchers from a wide array of fields, and its terminology needs to be flexible enough to accommodate both novel forms of usage and some degree of standardization in order to shape a lingua franca.

$\mathrm{QE}$ research involves or is conducted on Discourse data [1,2]; this may include data in the form of spoken or written speech (interview transcripts, log files, social media data, etc.), annotations on observations (field notes, structured observation, etc.), and visual data (video recordings, photovoice, etc.). Alongside discourse, other types of data may be collected as well. For example, when Shum et al. investigated teamwork in nursing team simulations, apart from the discourse of participants, they also recorded heart rate, skin conductance, movement, and (co-)location [3]. Furthermore, different 
forms of metadata may be collected, e.g., Dowell et al. were interested in how individual-level roles and group compositions influence both student and group performance during collaborative interactions in online collaborative-learning environments. Aside from analyzing discourse, they collected metadata such as which dataset and which student group the data provider belonged to, as well as assigned a timestamp and a unique identifier for each of their contributions [4]. Metadata can be used to group data providers and their discourse, enabling a wide variety of analyses.

In order to represent various types of data in a single, unified dataset in a human and machine-readable format, qualitative data needs to be quantified. This is typically achieved through discourse segmentation and coding. Critically, discourse data needs to be transcribed, which involves a number of decisions, e.g., spoken words are transformed into sentences including punctuation and diarization - assigning spoken language to given speakers. Segmentation, or dividing discourse data into meaningful parts, may occur on several levels. The lowest level of segmentation is referred to as an utterance; in text data these are usually a sentence or a turn-of-talk [2]. Utterances may be grouped into "stanzas" and "conversations" to provide relational or temporal context. Content within discourse is represented by applying inductively (bottom-up, grounded) or deductively (top-down, a priori) developed discourse codes [5]. In the final dataset, each row of the "qualitative data table" [2] contains an utterance and each column represents a variable with values in categorical or binary form, which can be metadata or discourse codes. This unified dataset can then be employed for performing various analyses and modelling complex interactions.

Several tools and techniques have been developed under the auspices of QE, aiming to facilitate a part of the process described above or to enable various analyses using the generated dataset. For example, nCoder is a platform that can be utilized to develop, refine, validate, and implement automated coding schemes. One aspect of code validation is ensuring raters share a common understanding of each discourse code they employ; this is generally achieved through coder training and Inter-Rater Reliability (IRR) and tests of agreement, such as Cohen's Kappa. Aside from IRR testing, nCoder can be employed for automated coding based on classifiers, an algorithmic process that identifies whether a piece of data belongs to a certain category or class [5]. This is helpful when working with large corpora, for example. Another QE tool, the Reproducible Open Coding Kit (ROCK) is a standard for working with qualitative data, helping researchers organize data sources, designate characteristics of data providers, as well as code and segment discourse data. The ROCK is implemented in an R package and a graphical user interface that eases manual coding of data. [6]

Epistemic Network Analysis (ENA) has been the flagship tool within QE, enabling researchers to explore and model salient patterns in their data with network graphs. ENA depicts the structure of connections among codes in discourse by calculating the co-occurrence of each unique pair of codes within a designated segment of data, and aggregates this information in a cumulative adjacency matrix. ENA represents this matrix as a vector in high-dimensional space, which is then normalized to quantify relative frequencies of co-occurrence independent of discourse length or simple volume of talk. In this process, network connection strengths are transformed to fall between zero and one, and ENA then projects the networks as points into a low-dimensional space using 
singular value decomposition (SVD), which maximizes the variance explained. This analytical tool hence provides two, coordinated representations of the unified dataset: 1) network graphs, where the nodes in the model correspond to the codes in the discourse and the edges represent the relative strength of connection among codes, and 2) ENA scores, or the position of the plotted points on each dimension of the plotted points for units of each network in the low-dimensional space. ENA uses two parameters to operationalize relational context for each unit of analysis: conversation and stanza window. Conversations are groupings of utterances, which can be connected in a model, and stanza windows are an option for defining how connection structure is computed within conversations for a given unit of analysis. Network models are thus constructed based on researcher decisions made when creating the qualitative data table (e.g., operationalization of segmentation and coding) and decisions regarding network parameterization (e.g., designation of unit, conversation, stanza window). [7]

Due to the fact that decisions in research design, data collection, data transformation and representation all potentially affect results, and by virtue, conclusions drawn from those results, it is crucial to make conscious choices throughout the entire process. Well-informed decisions are founded on individuals' access to the cumulative knowledge that a field has generated. Such knowledge is in part conveyed via reporting on vital features of the research process within publications. In the case of $\mathrm{QE}$, because it is still a developing methodology, reporting standards are yet to be solidified. Accumulated knowledge is also effectively conveyed through researchers taking advantage of Open Science (OS) practices, such as making their research process (codebook, analysis scripts, etc.) transparent via a public repository. OS principles and practices are gaining momentum in the scientific community, but primarily among initiatives involving quantitative methods. As a unified, quantitative - qualitative methodology, QE faces the challenges and affordances of both worlds regarding how OS principles may be implemented and what kind of practices may be developed.

As researchers aspiring to further refine QE methodology and to develop novel QE tools, we wanted to initiate a living (continuously ongoing) systematic review of the work that is being done in the $\mathrm{QE}$ community in order to see the choices we, as a community of scholars, have made in research design and operationalization. We were also interested in how QE scholars have conceptualized QE, how they have defined it for themselves and their audiences. We explored these topics in various $\mathrm{QE}$ works with the aim of 1) providing an overview of methodological decisions and potential choices one can make in a QE study, 2) shedding light on some challenges and opportunities of standardizing QE-related concepts and terminology, and 3) identifying areas where transparency may be beneficial for the community. We did not aim to reach final conclusions on methodological challenges or settle terminological debates; rather, we wanted to raise questions in these topics and explore some directions various $\mathrm{QE}$ authors have taken thus far. Furthermore, as we are performing a scoping review (i.e.: mapping out the literature without addressing substantive questions addressed by the literature), we were not attempting a meta-analysis of any sort, nor a deep evaluation of any aspect of research design (e.g.: sampling, validation) as that is outside the focus of this review. 


\section{Methods}

A more detailed description, including our decision-making processes, codebook, extraction template and other materials can be found in our public repository: https://osf.io/pa9jv/. This study was preregistered at Open Science Framework Registries.

\subsection{Entity Specification}

Our extraction process entailed coding for specific characteristics explicitly stated in the published works, from herein referred to as "entities". We developed a list of entities based on basic information constituting the citation and three guidelines: 1) Standards for reporting qualitative research: a synthesis of recommendations (SRQR; [8]) detailing general requirements for reporting in qualitative research, 2) The book Quantitative Ethnography [2] expounding fundamental aspects of QE-related research and specific features that can be considered and reported on in a QE study, and 3) Transparency standards covered by the Guidelines for Transparency and Openness Promotion in Journal Policies and Practices "The TOP Guidelines" ([9]). A complete list of our entities, their sources, and justifications for employing them can be found here: https://osf.io/tvudx. Following this, we carried out a pilot study on the proceedings of the First Conference on Quantitative Ethnography, which included the phases of coding subsets of our data, triangulating our results, and refining our code system. Our complete process is available here: https://osf.io/46xtg. Subsequent to our pilot [10], the code system was finalized. Our final, complete codebook can be found here: https://osf.io/avkbn.

\subsection{Search Strategy}

Since QE developed from the discipline of learning sciences, and more generally social science, we considered databases with the most content regarding these (ERIC, JSTOR). We also considered databases with content related to healthcare and psychology (PubMed and PsycINFO), as many recent QE publications have been in this subject. This database search was complemented with the open Zotero database of QE hub University of Wisconsin-Madison. Our query string is identical with that used in the construction of the Zotero database, containing the term $\mathrm{QE}$ and the main tools developed in QE (ENA and nCoder). For the complete query including logical operators, see: https://osf.io/cb9ag. We did not attempt to obtain gray literature, only peer-reviewed publications were included. 


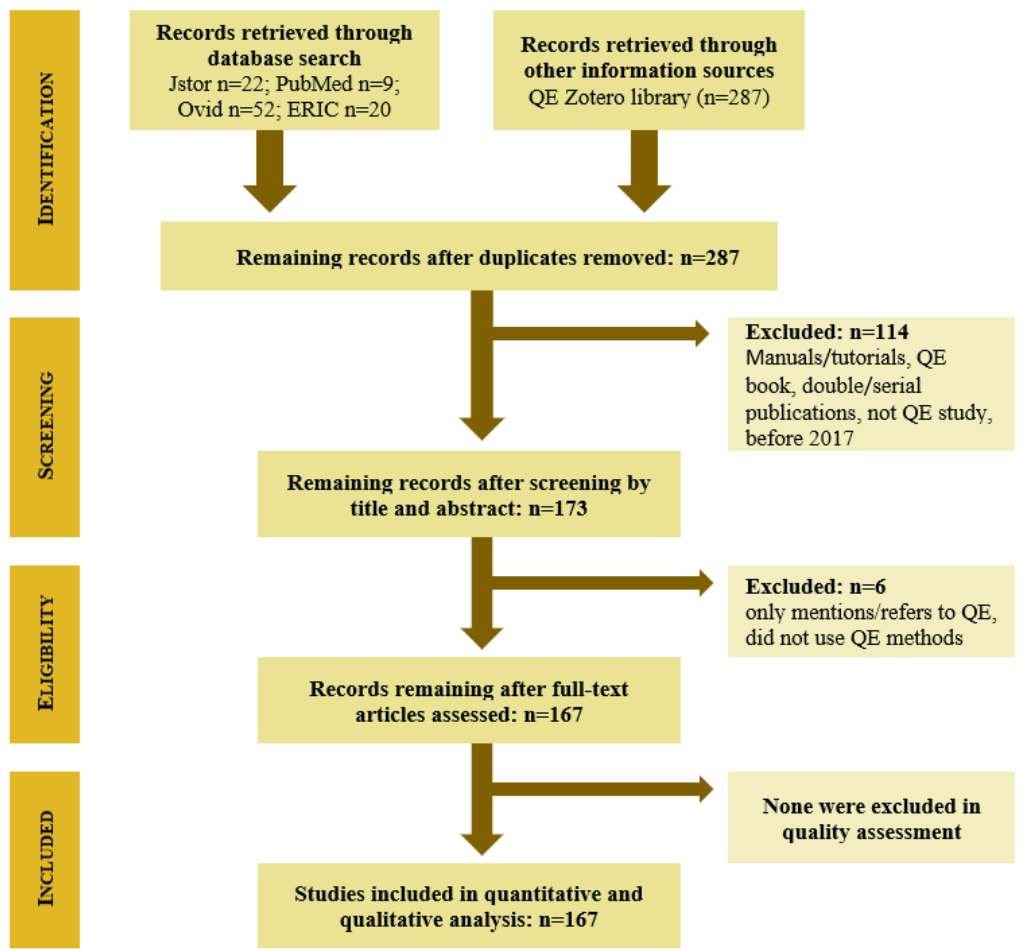

Fig. 1. PRISMA diagram depicting the screening and inclusion process of publications in Quantitative Ethnography following the year 2016. The present study contains 66 publications from the total.

\subsection{Screening and Study Selection}

The resulting hits were exported (2020/11/29) and screened by two independent screeners in one screening stage, which was followed by automatic duplication flagging. In the screening stage, we inspected titles, keywords, abstracts, and, if it was unclear whether to include or exclude a source based on that information, the full text was acquired and screened. We aimed to include all published works that report to have used QE methods in their initiative. Our query was highly inclusive, with very general terms and only limited to works in English language after 2016 and no specific geographical area. We excluded the book Quantitative Ethnography by Shaffer as we considered this as a methodological and temporal null point for QE-related publications; we wanted to see how the discipline has progressed since this seminal work, as conceptualized and operationalized by authors other than other than in this inaugural publication. Furthermore, we excluded duplicate and serial publications; all publications before the year 2017; and publications only mentioning or citing QE as a discipline or the tools nCoder and ENA (i.e. when these tools did not constitute the method of data collection or analysis). Figure 1 displays a PRISMA diagram of our screening process. 


\subsection{Data Extraction}

Extraction in both our pilot and current phase was performed by humans and extraction was completed for entities only if the information was explicitly stated within the publication. Pairs of coders worked together on a single source, then triangulated their results and resolved any differences through social moderation. Extraction forms (source code files for R) were completed using Notepad++ by specifying variable values in a template file (available here: https://osf.io/ekxtj). All extraction forms (from the four individual raters and the final version the pair generated during triangulation) can be viewed in our repository. The four raters met regularly to discuss the coding process and update code definitions; two other researchers provided validation for coding and verified extraction formatting. All authors of included studies were invited via the International Society for Quantitative Ethnography (ISQE) newsletter to fill out a Qualtrics survey, which contained the items of our systematic review. Authors have continuous access to our results at our repository, and are welcome to contact us if they see errors in interpretation.

\subsection{Statistical Analysis}

All completed R source code files were processed by an R script ('metabefor' available at: https://gitlab.com/r-packages/metabefor), with which we could perform computations and generate overviews.

\subsection{Thematic Analysis and Quantitative Content Analysis}

The entity "QE conceptualization and use" was a string that yielded a large amount of qualitative data. To transform this data into a more abstract form, two researchers, working autonomously, employed different analytical approaches on the same data. One researcher used Thematic Analysis [11], the other utilized a word frequency count to identify words of potential interest and then employed a Key Word In Context Search (KWICS) to test for the consistency of usage of words [12]. After that, they worked together to reach a consensus regarding common themes and trends.

\section{$3 \quad$ Results}

\subsection{Basic Information}

At this point, we have processed 66 publications in our systematic review, following an alphabetical order in publication title. The list of included studies can be accessed here: https://osf.io/anmrq. This list also comprises the extractions from our pilot study, so several publications are from the year $2019(n=45)$. As we process more publications included into our living review, we will be able to report on them; now we focus on the 66 extractions performed thus far, which constitutes a fair sample as study title can be considered a random mode of sampling.

Authors were from a total of 22 countries, there were 19 international collaborations where universities from at least two different countries were represented; the majority 
of authors were affiliated with universities from the United States $(n=39)$. In 22 instances, this information was not available in the full text of the publication. Data collection took place in a total of 17 countries; in 20 instances the origin of data was not specified, in 6 cases data was scraped from an international database. Most inspected studies were book chapters or papers in conference proceedings $(n=49)$, fifteen were journal articles, one was a dissertation. The majority of studies in our review addressed topics the learning sciences, specifically regarding collaborative learning, problemsolving, and computer-supported collaborative learning (CSCL); other significant fields include healthcare and identity research.

\subsection{Study Design}

The majority of studies were empirical ( $n=44)$, four were theoretical (no empirical data was collected), and 13 papers explicitly explored theoretical issues with empirical data obtained in a previous study. Empirical studies were conducted on data from human participants, but sample size did not always indicate the number of human participants, sometimes it referred to their contributions in an observational setting, posts on social media, or scientific outputs in archival research.

Although the sample was described to some degree in most studies, sampling considerations were rarely reported; in 11 instances absolutely no information was given on sampling. Sample sizes ranged from 1 to 2508 ; twenty-four were between 2 and 50, eleven were between 51-100, and eighteen were over 100. There were 5 case studies. In eleven instances, sample size information was not reported in the study. Twentythree studies regarded the individual participant as the source of data; five studies collected data from groups of participants, and twenty-one publications examined data from both individuals and groups. In a total of three studies, data was collected from dyads of participants. The most frequent forms of collected metadata were: participant age, sex, education, location, and timestamps for utterances.

The most common form of data was text $(\mathrm{n}=36)$, followed by audio or audio-visual data $(n=20)$, and numerical data $(n=4)$. In five cases, the type of data gathered was not reported by the authors. Data were collected with a variety of methods, such as making observations in-person or asynchronously $(\mathrm{n}=24)$, using an archive (pre-existing archive or database) to retrieve data $(\mathrm{n}=21)$, gathering data from synchronous chatroom interactions ( $\mathrm{n}=12)$, administering surveys $(\mathrm{n}=10)$, conducting semi-structured or structured interviews $(\mathrm{n}=9)$, and leading focus group discussions $(\mathrm{n}=2)$. In eighteen studies a combination of these were employed, and in eleven instances other methods were used to collect data. In two publications authors either failed to report the method of data collection or did not explain it sufficiently for us to perform extraction. The most common data collection method and data type were text retrieved from an archive and audio-video data from observation. Figure 2 shows a heatmap of co-occurrences for data collection method and data type among the publications reported on in this study. 


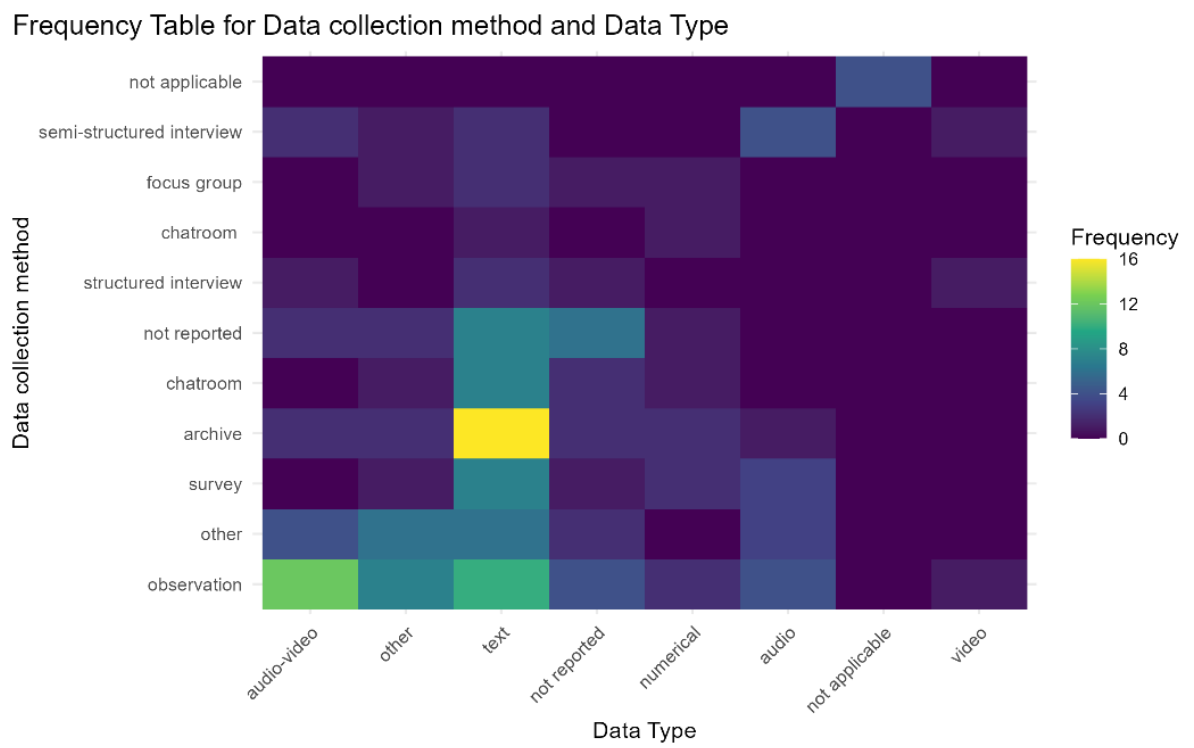

Fig. 2. Heatmap of co-occurrences in values for extracted entities Data collection method and Data type among the 66 publications reported on in this study. Colors represent the frequency of co-occurrence; yellow displays the highest, dark blue the lowest frequencies.

\subsection{Coding}

Most researchers employed a combination of deductive and inductive coding $(n=20)$ or solely deductive coding $(n=21)$; a small proportion of researchers used inductive coding $(n=8)$. In the case of seven studies, coding was not performed, and eight publications did not report what type of coding was used. Ten studies did not report any considerations in how codes were developed or where they were adopted from. The number of employed codes ranged from 2 to 52; thirty studies used 1-10 codes, fifteen used 11-20 codes, and six had more than 20. Sixteen publications did not report the number of codes they employed or the publication did not contain sufficient information for extraction. The majority of studies used both automated coding (machine performs coding autonomously based on e.g. regular expressions) and manual coding (human performs coding in some form of annotation; text coded by hand) $(n=17)$, or just manual coding $(n=19)$; five publications used automated coding only. Authors of fourteen studies failed to explicitly state how they coded their data. The most common coding type and coding process were manual deductive coding and deductive coding in a process that was not reported. Figure 3 shows a heatmap of co-occurrences for coding type and coding process among the publications reported on in this study. 


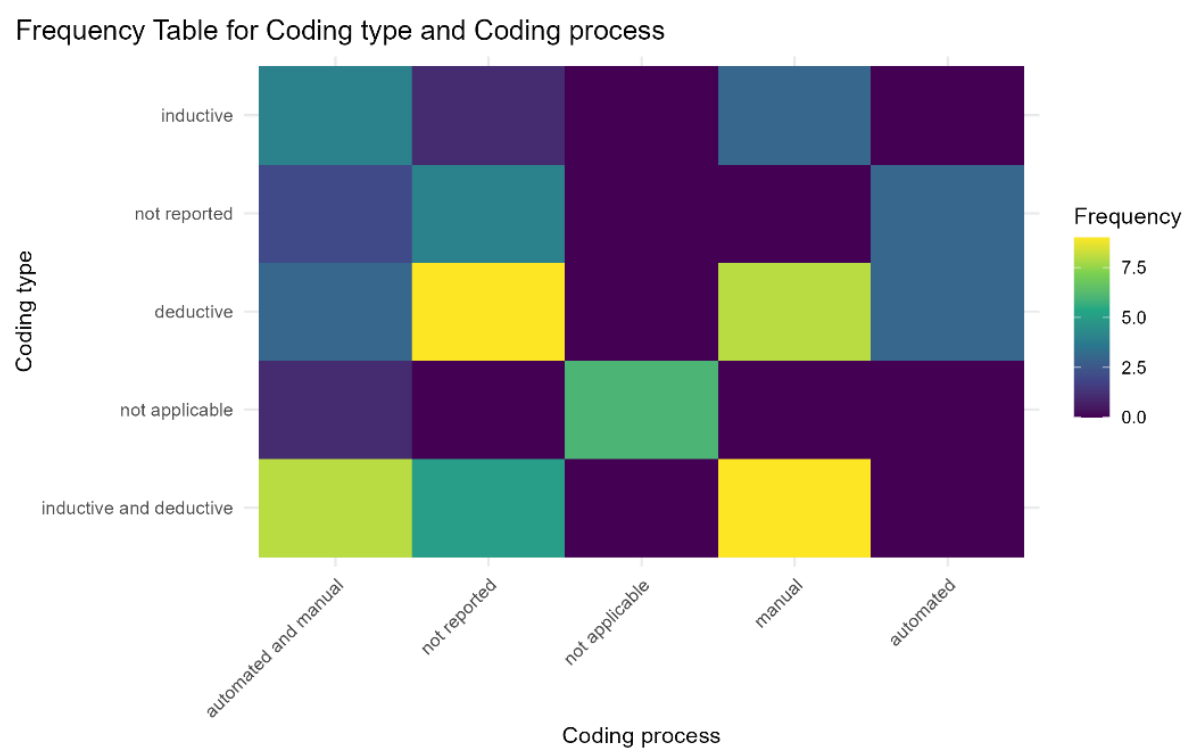

Fig. 3. Heatmap of co-occurrences in values for extracted entities Coding type and Coding process among the 66 publications reported on in this study. Colors represent the frequency of cooccurrence; yellow displays the highest, dark blue the lowest frequencies.

Coding was performed by human raters in most studies $(n=23)$, usually two researchers working autonomously $(n=19)$; in nineteen cases both humans and a computer coded data, and in one case fully automated coding was used. In fifteen studies, authors did not report whether the data was human- or machine-coded. Of the publications where this was applicable, the vast majority calculated IRR $(n=31)$.

\subsection{Segmentation}

Discourse segmentation was applicable in 54 studies, twenty-nine of those performed segmentation and reported it, twenty-five did not report this in their publication. The smallest unit of segmentation (i.e., utterance) was most frequently operationalized as "a line of data"; it was not always clear what a "line" constituted or how it became "a line". Following this, utterances were most commonly defined as a "turn-of-talk", a sentence, a response, or a second in time. Less concrete operationalizations included a "comment", a "chunk", "a line which could contain a dialogue or an event", "lines of explanation", an "interaction", and "one chat". In eleven studies where this was applicable, authors did not report their operationalization of utterance. The majority of studies conflated mid-level segmentation (stanza) with model parameterizations (stanza window, conversation), and most frequently defined stanza with a moving stanza window of 3-7 utterances $(n=14)$. Others defined stanza as: steps in a process, phases in a task, lesson plans, topics, questions from the interview guide and their follow-ups, or online "boards" teachers use to post material for students. Where utterances were periods of time, stanzas were a certain number of lines, e.g.: 20 lines of data representing 
4.4 seconds during which a conversation exchange takes place, or 10 lines of data corresponding to 20 seconds of gameplay. In eleven instances, where it would have been applicable, authors did not explicitly state their operationalization of stanza.

\subsection{Analysis}

Fifty of the 66 publications generated network models with ENA. Most frequently, individual participants constituted the unit of analysis $(n=13)$, in a singular state or multiple states at different points in time. Units were also commonly operationalized as groups of participants $(\mathrm{n}=12)$. Additionally, phases within an activity (stages, steps, or conditions; $n=8$ ) or an entire activity (lesson plan, session; $n=3$ ) were considered units. Less frequently, units were defined with metadata (e.g., age of participant, date of data collection), or as timestamps, interactions, outputs that individuals or groups created, or a "turn-of-talk". Sometimes authors employed a combination of these to operationalize unit, such as an individual in a phase of an activity. In instances where networks were created, and thus the designation of units was applicable, seven studies did not report their decision. Apart from ENA, other analytical methods, techniques, and tools were employed, such as Process Mining, Sequential Pattern Mining, Quantitative Multimodal Interaction Analysis, Non-negative Matrix Factorization, Agglomerative Hierarchical Clustering, Logistic Regression, Independent Samples t-Test, Two-sample Mann-Whitney U Test, Principal Component Analysis, Latent Semantic Analysis, Group Communication Analysis, Latent Dirichlet Allocation, Bartlett's Test of Sphericity, and Socio-Semantic Network Analysis.

\subsection{Conceptualization}

Six papers in our sample did not contain any discussion or description of ENA or QE conceptualization and reasons for it use. Those who did choose to include descriptions centered the conversation primarily around ENA, rather than QE. In fact, almost all references to QE were to home ENA as the main methodology, or to simply cite Shaffer's book, Quantitative Ethnography. Essentially, ENA was used to define QE, and QE was used to define ENA. Even in descriptions of QE independent of ENA, the definition of QE bore close similarity to that of ENA, centering the conversation around the types of data handled by QE: quantitative and qualitative, big data. Not only are ENA and QE often conflated as means for conducting research, there is also an uncertainty in whether QE is a novel field, a methodology, or a tool. Most descriptions of ENA were focused on describing the main elements involved in ENA (data, codes, units, networks) and its functionality (analysis, visualization, modelling connections). While some papers dived deeper into the complexities of ENA, describing dimensional reduction, projection spaces, temporal context, and the types of data supported, others simply defined ENA as a tool or an algorithm. Finally, some papers explicitly described how ENA was applicable to their data analysis, referencing topics like identity exploration, collaborative learning, and qualitative analysis more broadly as phenomena they aimed to investigate when using ENA on their data. 


\section{Discussion}

Our living systematic review aims to provide an overview of choices that the QE community has made in research design and how scholars working with this methodology have conceptualized QE. Below we briefly summarize our results, place them in a greater context, and address challenges and opportunities of articulating QE-related concepts, while pinpointing areas where transparency might aid the development of QE methods.

Discourse Equals Text? QE methods are applicable to a range of discourse data, but the majority of studies in our sample involved text data, most commonly from observations, archives, chatroom discussions, or interviews. A scoping review by Kaliisa et al. [13] examining the boundaries, opportunities, and challenges of QE analyzed 60 papers, similarly noting that most QE studies worked with text-based raw data and that non-text data (e.g., videos) were always converted to text. This points to a facet of QE methodology where, albeit raw discourse data can be audio or visual in nature, in order to perform analyses, it must be transformed, most commonly transcribed, which involves a series of crucial decisions. Some discourse data may come in a more "curated" form than others, e.g., log files from a virtual collaborative game. Yet in other instances, where audio constitutes raw data, such as in the case of interviews, transcription involves making decisions, such as where to place what punctuation mark, assigning speech segments to participants, etc. When non-text data is utilized, the number of these decisions may be higher and more complex, such as in the case of recording observations. Data is hence constructed and its transformations affect coding [14]. For example, when coding interviews whether on the level of sentences of question-response, the frequency and co-occurrence of codes are potentially influenced by how the spoken utterances were transcribed. In another example, raw video data is coded based on how a series of pictures is transformed into categories describing the content of that visual data (e.g.; spoken speech, speech acts, relational location). Our results indicate that the majority of QE researchers worked with text data, only a small portion worked with raw data that was not initially text, but even those data were transformed/transcribed into text and then processed further. In QE, discourse data is most easily imagined and most commonly employed as text, visual manifestations of discourse are yet to be thoroughly explored within the QE paradigm.

Should Sampling Be an Issue? In several instances, authors did not report considerations in sampling, which may stem from the fact that many studies employed qualitative methods for data collection and part of analysis as well. Traditionally, in qualitative studies, it is not easy to calculate ideal sample size and researchers often utilize convenience sampling with no major guiding criteria regarding whom to include in the study. If QE asserts being a unified methodology, with the ability to make both qualitative and quantitative claims, should there be more emphasis on sampling in QE studies? Kaliisa et al. note that 44 out of 60 reviewed QE studies had a sample size of less than 100, and argue that this "small sample size" can lead to challenges in drawing conclusions and integrating statistical approaches, as well as affect the application of 
QE at scale [13]. One could also argue that this is not an issue of sample size, but of sampling: if the sample was not chosen at random from the target population, then it is the sampling procedure that prevents generalization to the wider population, not the size of the sample. Should random sampling be an expectation for QE initiatives, though? In other words, how highly does QE prioritize external validity compared to other desiderata? Also, if data collection was not systematic (e.g. it adapted flexibly to the data collection process itself or to psychosocial circumstances, as in the case of semi-structured interviews, for example), that will generally deviate from the assumptions regarding sampling distribution variance required by most quantitative methods. That inability to model error terms may prevent estimation and inference. Again, this is not a problem that can easily be solved by increasing the sample size; and it remains to be established how problematic this is for the most common QE analyses (e.g. ENA) and more widely, how highly internal validity is prioritized. Additionally, there are different types of validity and generalization as well as different criteria for power analyses and sample sizes. Beyond the mechanics of statistical methods, there are epistemic considerations of what constitutes a "fair sample" [15], which in turn depends on what it is a sample for (what analyses, conclusion and inferences) and who it is fair to.

What Constitutes the Sample? Many studies in our review report sample size, but it is unclear what or whom they consider as constituting N. In instances, authors may report a sample that consists of outputs from individuals or groups, such as drawings from children in an art class. Since each child can make several drawings, it is worth explicitly stating which of these entities constitutes the sample, e.g. the children or their drawings. Both of these can constitute the N, and as QE methods accommodate investigating both or either one, this may warrant a terminological distinction. One solution may be differentiating between a case (data provider, e.g. a child) and a source (bounded piece of discourse to be coded, e.g. a drawing); this would enable a clearer reporting of what is considered the sample and how it can be described. Additionally, being transparent about how the data is handled in e.g. the coding phase, would be useful to understand research design and generally the results. For example, data might have been collected from individual students working in dyads or teams in a classroom activity, but how that data is subsequently handled may differ: discourse from the group may be aggregated prior to coding or individual contributions may constitute autonomous units of analysis; there may be aspects of analysis that pertain to the entire group (e.g., evaluation of a collaborative exercise based on some set of criteria) and to an individual (e.g., style of collaboration based on a taxonomy).

Distinctions in Metadata? In QE studies, discourse constitutes data, and other information about data is referred to as "metadata". Metadata is used to group data providers and/or utterances in order to create various models via conditional exchangeability. [2] Authors in our review most frequently designated age, sex, time, and location as metadata. Some authors (e.g. [16]) have pointed out the interchangeability that exists between metadata (grouping variables; categorical) and discourse codes (binary representation). Perhaps making a distinction between data about data (metadata) and data about data providers (attributes) is a useful one, as aggregation and analyses may 
benefit from differentiating between, on the one hand, a timestamp on an utterance or the date of an interview, and on the other hand, a characteristic of a participant, such as age, sex, level of education. Another distinction might include a way of indicating what is (potentially) considered both a discourse code and a grouping variable in a particular study.

Why be Open about Coding? Coding entails a process of defining relevant and meaningful aspects of a discourse and locating them within the data. Shaffer and Ruis argue that a set of coded data should be able to make fair claims about some set of codes for each of its segments [14]. The concept of fairness they introduce is founded upon providing definitions and examples for the employed codes, i.e. a codebook. These codes can be developed inductively (grounded, emergent, bottom-up) or deductively (a priori, theoretical, top-down); reporting the process by which codes were created or from where they were adopted is a crucial aspect of validity and accountability [5, 14]. Whereas Kaliisa et al. found that most authors were using deductive coding, our sample included studies that were using a combination of both. This may be further complicated by the fact that a coding process may include both an inductive and a deductive phase, but authors may only report on one of these, e.g. how they inductively developed the codes or in what way they deductively applied them to the corpus. In our sample, codes were often adopted from a theoretical framework, such as Technological Pedagogical Content Knowledge (TPACK) describing the kinds of knowledge necessitated by teachers, or Projective Reflection (PR), a theory of learning commonly used in QE literature on identity construction and trajectories. In such cases, using open codebooks that incorporate classifiers employed in deductive and/or automated coding would benefit all authors who subsequently wish to use this framework, as their list of classifiers would be enriched by the existing pool.

How Was IRR Verified? We investigated the frequency of QE scholars employing a common measure of agreement between raters, IRR. Of the 66 studies included where IRR would have been applicable, about half calculated it, usually via Cohen's Kappa, and about half did not report whether this was performed. Kaliisa et al. coded for various types of code validation, but arrived to similar conclusions $(n=23 / 60)$, adding that many authors did not report the exact statistical procedure applied to compute IRR or did not disclose other forms of validation [13].

Does Segmentation Differ from Model Parameterization? Our results show that the operationalization of various levels of segmentation can differ greatly. For example, a turn-of-talk (e.g., a Twitter post/reply) can be one researcher's utterance and another's unit. In some cases, only the lowest level of segmentation was defined, e.g. "lines of data" with no evident operationalization of mid- or high-level segmentation. Often, how segmentation was achieved was unclear: what steps were taken to curate the data in this way and why? Some data may be generated or collected in a way that automatically creates some type of segmentation, such as threads in social media data or "boards" used in virtual educational environments. Are these "naturally" occurring types of segmentation the most optimal to answer the research questions authors have? How can 
we determine the granularity needed for our investigation? Furthermore, mid-level segmentation (stanza) was often conflated with model parameterizations, where a stanza was defined by how code co-occurrences were accumulated in ENA models (stanza window) or how utterances were grouped (conversation). Whereas often these may coincide, there is a conceptual distinction among them. Formulating justifications for these decisions and reporting on them (or creating supplementary materials) could be beneficial not only for the authors but the QE community as well.

Is QE Fully Described by ENA? As Kaliisa et al. also note, an overwhelming majority of QE scholars employ ENA to model and analyze their data. Our results indicate that other statistical and qualitative methods are also being used to complement ENA, but do those methods constitute part of QE? How can we define QE and distinguish it from other methods, methodologies? Many scholars conceptualize QE with ENA; what are the implications of defining a methodology with a (single) tool? Kaliisa et al. simultaneously call for scholars to place QE principles at the center of their research design, but also assert that the QE community is yet to define standards that delineate QE methodology [13]. This may be because if a methodology is in such close dialectic with a tool, it is difficult to separate the two. QE can be described as a unified methodology, accommodating the scrutiny of many different types of raw data, which are coded and segmented in an automated or manual process with validated codes, and aggregated in a dataset that systematically captures both qualitative and quantitative aspects of data in a human and machine-readable way. What is specific to ENA about these tenets and what other tools can be developed along these lines? As QE matures, other tools will be needed to both implement and enhance our understanding of a unified methodology.

Does Reporting Exhaust Being Transparent? The lack of reporting of various methodological decisions may have several explanations: authors might have spatial limitations, may not believe it is necessary, their decisions may not always be conscious, and so on. Yet, much can be gained from making research processes transparent, not only for the authors themselves (logging decisions, justifications, rationale for future reference), but also for a community of scholars facing similar methodological challenges or trying to interpret others' results. Transparency allows the pooling of knowledge, not only regarding best practices and clever solutions to problems, but also the conundrums themselves, and even decisions that did not work well in the end. Our results indicate that authors generally did not disclose materials generated in their initiative, although, in a few instances, authors would share a URL to a complete codebook, but it would take the viewer to an inaccessible page (e.g., Google doc needing authorization to view). This demonstrates that even if a scholar agreed with the principle of providing others with an opportunity to scrutinize processes in more detail, they may be lacking information on how to share materials generated in their research process in a FAIR (findable, accessible, interoperable, and reusable) manner. 


\section{$5 \quad$ Limitations}

Our review had several limitations. Concerning the applied methodology, the specified entities to extract were developed by a relatively small group of researchers, albeit we conducted a pilot study to explore the developed entities, as well as other possible values and entities. Regardless, we selected relatively few entities for pragmatic reasons; although, these can be expanded upon in the coming iterations of the living review. Furthermore, categorizations within each entity were not pretested for validity. Also, our present entities need further specification, for example, we need to develop separate values for papers and posters in conference proceedings, as spatial affordances are vastly different in various forms of submissions, and this may influence reporting in manuscripts to a large extent. Also, we need to account for different forms of code validation, not only IRR, as this is a crucial tenet of QE but it is presently limited to one validity measure. Additionally, because QE hub, University of Wisconsin-Madison is over-represented in our sample, the "mirror" provided by this review is highly expressive regarding work conducted at the Epistemic Analytics Lab. Also, some regions dominated the sample, namely the US, Australia, and Europe, and at present it is not possible to draw conclusions on whether our results reflect QE in these regions or QE in general.

\section{Closing Remarks}

Although initially emerging from the learning sciences, QE is now situated at the nexus of multiple disciplines; and it is currently answering the challenge of establishing a lingua franca that is sufficiently specific, yet remains plastic enough for usage in vastly different research settings. Our analysis intends to spur discussions on methodological and terminological issues within the community with the aim of enhancing transparency and rigor.

\section{Acknowledgements}

This work was funded in part by the National Science Foundation (DRL-1661036, DRL-1713110), the Wisconsin Alumni Research Foundation, and the Office of the Vice Chancellor for Research and Graduate Education at the University of WisconsinMadison. This project has also received funding from the European Union's Horizon 2020 research and innovation program under the Marie Sklodowska-Curie grant agreement No. 101028644. The opinions, findings, and conclusions do not reflect the views of the funding agencies, cooperating institutions, or other individuals.

\section{References}

1. Gee J (2014) An Introduction to Discourse Analysis: Theory and Method. Routledge, London 
2. Shaffer D (2017) Quantitative Ethnography. Cathcart Press

3. Buckingham Shum, Simon, Echeverria, Vanessa, Martinez-Maldonado, Roberto (2019) The Multimodal Matrix as a Quantitative Ethnography Methodology. In: ICQE 2019: Advances in Quantitative Ethnography. Springer, Madison, WI, pp 26-40

4. Dowell N, Nixon T, Graesser A (2018) Group Communication Analysis: A Computational Linguistics Approach for Detecting Sociocognitive Roles in Multi-Party Interactions. Behavior Research Methods 51:1007-1041. https://doi.org/10.3758/s13428-018$1102-\mathrm{z}$

5. Cai Z, Siebert-Evenstone A, Eagan B, et al (2019) nCoder+: A Semantic Tool for Improving Recall of nCoder Coding. In: ICQE 2019: Advances in Quantitative Ethnography. Springer, Madison, WI, pp 41-54

6. Zörgő S, Peters G (2019) Epistemic Network Analysis for Semi-Structured Interviews and Other Continuous Narratives: Challenges and Insights. In: Advances in Quantitative Ethnography. Communications in Computer and Information Science Series. pp 267-277

7. Bowman D, Swiecki Z, Zhiqiang C, et al (2021) The Mathematical Foundations of Epistemic Network Analysis. In: Advances in Quantitative Ethnography. Communications in Computer and Information Science Series., Eds. Ruis AR and Lee, SB. Springer Nature, Switzerland, pp 91-105

8. O'Brien BC, Harris IB, Beckman TJ, et al (2014) Standards for reporting qualitative research: a synthesis of recommendations. Acad Med 89:1245-1251. https://doi.org/10.1097/ACM.0000000000000388

9. Nosek B, Alter G, Banks G (2020) Transparency and Openness Promotion (TOP) Guidelines

10. Porter C, Donegan SR, Eagan B, et al (2021) A Systematic Review of Quantitative Ethnography Methods. In: ICQE 2020: Conference Proceedings Supplement. ISQE, Virtual, pp 35-38

11. Bazeley P (2013) Qualitative data analysis: Practical strategies. Sage Publications, Thousand Oaks, California

12. Stemler S (2000) An overview of content analysis. Practical Assessment, Research, and Evaluation 7:

13. Kaliisa R, Misiejuk K, Arastoopour G, Misfeldt M (2021) Scoping the Emerging Field of Quantitative Ethnography: Opportunities, Challenges and Future Directions. In: ICQE 2020: Advances in Quantitative Ethnography. Springer, Virtual, pp 3-17

14. Williamson Shaffer D, Ruis A (2021) How We Code. In: ICQE 2020: Advances in Quantitative Ethnography. Springer, Virtual, pp 62-77

15. Williamson Shaffer D, Serlin R (2004) What Good Are Statistics That Don't Generalize? Educational Researcher 33:14-25. https://doi.org/10.3102/0013189X033009014

16. Misiejuk K, Scianna J, Kaliisa R, et al (2021) Incorporating Sentiment Analysis with Epistemic Network Analysis to Enhance Discourse Analysis of Twitter Data. In: ICQE 2020: Advances in Quantitative Ethnography. Springer, Virtual, pp 375-389 